\title{
Dynamical instability of a dark soliton in a quasi-one-dimensional Bose-Einstein condensate perturbed by an optical lattice
}

\author{
N.G. Parker ${ }^{\dagger}$, N.P. Proukakis ${ }^{\dagger}$, C.F Barenghi ${ }^{\ddagger}$, and C.S. \\ Adams $^{\dagger}$ \\ $\dagger$ Department of Physics, University of Durham, South Road, Durham DH1 \\ 3LE, United Kingdom \\ $¥$ School of Mathematics and Statistics, University of Newcastle, Newcastle upon \\ Tyne NE1 7RU, United Kingdom \\ E-mail: n.g.parker@durham.ac.uk
}

\begin{abstract}
The motion of a dark soliton is investigated in a one-dimensional dilute Bose-Einstein condensate confined in a harmonic trap and an optical lattice. The harmonic trap induces a dynamical instability of the soliton, culminating in sound emission. The presence of the optical lattice enhances the instability, and in addition, dephases the emitted sound waves, thus preventing stabilisation of the soliton by sound reabsorption. This instability can be probed experimentally by monitoring the soliton oscillations under various lattice configurations, which can be realised by changing the intensity and angle between the laser beams that form the lattice. For short enough times, such that the emitted sound does not reinteract with the soliton, the power emitted by the soliton is found to be proportional to the square of the local soliton acceleration, which is in turn proportional to the deformation of the soliton profile.
\end{abstract}

PACS numbers: $03.75 . \mathrm{Lm}, 42.65 . \mathrm{Tg}$ 


\section{Introduction}

The realisation of dilute atomic Bose-Einstein condensates (BEC) in multi-dimensional optical lattices has opened the door to study novel quantum effects within a pure and highly tunable system. These include the quantum phase transition from superfluid state to Mott insulator [1], interference of coherent matter waves [2, Bloch oscillations [3] number squeezed states 4], and arrays of Josephson junctions [5]. Following the recent experimental creation of dark solitary waves in harmonically trapped BEC [6, [7, 8] it is a now a realistic prospect to consider these macroscopic excitations in an optical lattice geometry.

A dark soliton is a one-dimensional localised wavepacket consisting of a notch in the ambient density and a phase slip across its centre. It is supported in repulsive/defocussing nonlinear media where the shape of the wavepacket is preserved by a balance between dispersive effects and nonlinearity. Dark solitons have been investigated thoroughly, both experimentally and theoretically, within the analogous field of nonlinear optics 9. However, the nature of trapped BEC poses new questions for the dynamics and stability of dark solitons. Thermal instabilities, arising from interaction with the thermal cloud, dissipate energy from the soliton, causing it to accelerate to the edge of the condensate and ultimately disappear [10, while slow solitons are sensitive to quantum fluctuations [11].

A dark soliton experiences two kinds of dynamical instabilites. Firstly, a dark soliton, which is strictly a one-dimensional object, is prone to transverse instabilities 12, 13, 14 when embedded in a two or three-dimensional system. For example, dark solitary waves under weak transverse confinement have been observed to bend via the "snake" instability and ultimately decay into vortices, in both BECs [15] and nonlinear optics [16]. However, by employing tight transverse confinement to freeze out the transverse degrees of freedom, quasi-one-dimensional condensates can be created 17, 18, 19, 20, in which dark solitons are expected to have greatly enhanced lifetimes [21]. The second dynamical instability arises from the fact that a dark soliton is a solution of a homogeneous system. The longitudinal confinement featured in BEC experiments breaks the integrability of the system, rendering the soliton unstable to motion through the inhomogeneous background density, and leading to decay via sound emission [22, 23]. In previous work we studied this decay mechanism within a harmonic geometry, and isolated two limiting cases. If the emitted sound can escape the system, the power radiated by the dark soliton is proportional to the local soliton acceleration squared 24, which in turn is intimately linked to the deformation of the soliton profile caused by the emitted sound waves [25]. In the opposite case of an infinite harmonic system, sound reabsorption occurs and the soliton decay becomes fully stabilised. To control this experimentally, we suggested creating a soliton in a tight dimple trap within a weaker harmonic trap. In such a geometry, the depth of the dimple (relative to the chemical potential) provides a sensitive handle on the solitonsound interactions, which can be observed experimentally by monitoring changes in the soliton amplitude and period [24.

Recent work by Frantzeskakis et al. 26, 27, has discussed the dynamics of slowlymoving dark solitons within optical lattices, including the effects of sound emission. The entire range of lattice periodicities were considered in [27, and, where appropriate, analytic models for the soliton dynamics were derived. In our work we perform detailed quantitative measurements of the sound emission from the soliton and show that the acceleration squared law discussed above is valid (prior to reabsorption of sound). We 
concentrate on much faster solitons than [27], and discuss in detail a range of lattice periodicities that can be readily achieved experimentally, where the lattice wavelength is comparable to, or slightly larger than, the soliton width. Given the number of current BEC experiments featuring optical lattices [1, 2, 3, 4, 5, 28, and the rapid soliton decay that can be induced in certain limits, this system may be preferential to investigate soliton-sound interactions than the dimple trap proposed in 24].

In Section 2 we introduce the system under consideration, the basic properties of a dark soliton, and the Gross-Pitaevskii equation used to simulate the soliton dynamics. In Section 3 we illustrate the dynamical behaviour of a dark soliton in an optical lattice, in particular the emission of sound, and compare this to the behaviour in the absence of the optical lattice. The dependence of the soliton dynamics on the lattice height and periodicity is discussed in Sections 4 and 5, and in Section 6 we analyse in detail the soliton dynamics and quantify the power radiated. Finally, in Section 7, we make some concluding remarks.

\section{Theory}

The dynamics of a trapped atomic BEC at low temperatures can be well described by the mean-field Gross-Pitaevskii (GP) equation. Assuming a quasi-one-dimensional condensate (oriented along the $x$-axis), the GP equation reduces to its one-dimensional form,

$$
i \hbar \frac{\partial \psi(x, t)}{\partial t}=\left(-\frac{\hbar^{2}}{2 m} \frac{\partial^{2}}{\partial x^{2}}+V(x)+g|\psi(x, t)|^{2}-\mu\right) \psi(x, t) .
$$

where $\psi(x)$ is the mean-field order parameter, $m$ is the atomic mass, and $\mu=n g$ is the chemical potential of the effectively one-dimensional system. The nonlinear coefficient $g$ arises from the interatomic interactions and has an effective one-dimensional form, $g=2 \hbar^{2} a /\left(m l_{\perp}^{2}\right)$, where $l_{\perp}=\sqrt{\hbar /\left(m \omega_{\perp}\right)}$ is the transverse harmonic oscillator length and $a$ is the three-dimensional $s$-wave scattering length. Repulsive interactions, $g>0$, are generally necessary to support a dark soliton, and will be assumed from now on. However, dark soliton-like structures can, under appropriate conditions, be supported by attractive interactions [29].

We consider an external potential of the form,

$$
V(x)=\frac{1}{2} m \omega_{x}^{2} x^{2}+V_{0} \sin ^{2}(2 \pi x / \lambda) .
$$

The first term represents a harmonic magnetic trap with frequency $\omega_{x}$ and the second term denotes an optical lattice with height $V_{0}$ and periodicity $\lambda / 2$, where $\lambda$ is determined by the wavelength and relative angle between the laser beams forming the lattice. Figure 1 shows a schematic of the trap configuration and initial condensate density.

On a uniform background $n_{0}$, the wavefunction of a dark soliton with speed $v$ and position $(x-v t)$ is given by,

$$
\psi(x, t)=\sqrt{n_{0}} e^{-i(\mu / \hbar) t}\left(\beta \tanh \left[\beta \frac{(x-v t)}{\xi}\right]+i\left(\frac{v}{c}\right)\right) .
$$

where $\beta=\sqrt{1-(v / c)^{2}}$ and $\xi=\hbar / \sqrt{\mu m}$ is the condensate healing length corresponding roughly to the size of the soliton 30. The soliton speed $v$ depends on its depth $n_{d}$ relative to the background density and the phase slip $S$ across its centre via $v=\sqrt{n_{0}-n_{d}}=c \cos (S / 2)$, with the maximum speed set by the Bogoliubov 


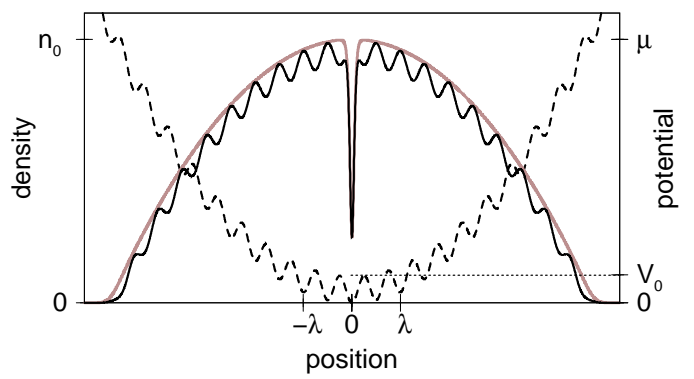

Figure 1. Schematic of the initial condensate density (solid bold line), in relation to the peak condensate density $n_{0}$, under the confinement of a harmonic trap and optical lattice (dashed line), with a non-stationary soliton located at the origin. The optical lattice has height $V_{0}$ and wavelength $\lambda$. The corresponding density in the absence of the optical lattice is also illustrated (solid grey line).

speed of sound $c=\sqrt{\mu / m}$. The renormalised soliton energy (after subtracting off the background fluid contribution) is given by 9 ,

$$
E_{s}=\frac{4}{3} \hbar n_{0}^{3 / 2}\left[1-\left(\frac{v}{c}\right)^{2}\right]^{3 / 2} .
$$

A stationary dark soliton reaches zero density $\left(n_{d}=n_{0}\right)$, features an abrupt $\pi$ phase slip, and maximum energy, whereas a soliton with maximum speed $c$ has no phase or density contrast, and therefore zero energy. Note that, to first order, a dark soliton behaves dynamically like a classical particle 31, 32.

Throughout this paper we employ harmonic oscillator units, where length is measured in terms of the harmonic oscillator length $l_{x}=\sqrt{\hbar /\left(m \omega_{x}\right)}$ and time in units of $\omega_{x}^{-1}$. In addition a fixed harmonic trap is always considered, corresponding to $\mu=70 \hbar \omega_{x}$, and simulations are performed for fixed chemical potential. We will assume realistic experimental parameters: $\omega_{x}=2 \pi \times 5 \mathrm{~Hz}, \omega_{\perp}=250 \omega_{x}$, and $\mu_{3 D}=8 \hbar \bar{\omega}$, where $\bar{\omega}=\left(\omega_{x} \omega_{\perp}^{2}\right)^{1 / 3}$. Then, for a ${ }^{87} \mathrm{Rb}\left({ }^{23} \mathrm{Na}\right)$ condensate, our space and time units correspond to $l_{x}=4.8(9.4) \mu \mathrm{m}$ and $\omega_{x}^{-1}=0.03 \mathrm{~ms}$. Furthermore these parameters roughly relate to a healing length $\xi=0.58(1.13) \mu \mathrm{m}$, speed of sound $c=2.5(1.3) \mathrm{mm} / \mathrm{s}$, one-dimensional peak condensate density $n_{1 D}=2 \times 10^{7}\left(5 \times 10^{7}\right) \mathrm{m}^{-1}$, and total number of atoms $N=4000$ (18000). All effects considered in this paper manifest themselves on timescales smaller than the $1 \mathrm{~s}$ anticipated soliton thermodynamic lifetime [10].

In order to realistically probe a range of optical lattice periodicities, we envisage employing two almost co-propagating laser beams, of wavelength $\lambda_{\text {laser }}$. By varying the angle between the beams $\theta$, the effective lattice wavelength can be increased beyond the actual laser wavelength, $\lambda \rightarrow \lambda_{\text {laser }} / \sin \theta$. Assuming a laser wavelength $\lambda_{\text {laser }} \sim 0.8 \mu \mathrm{m}$ (typically produced by a diode laser), then lattice wavelengths up to the order of $\lambda \sim 20 \mu \mathrm{m}$ can easily be produced, corresponding to $\lambda \sim 5 l_{x}$ for ${ }^{87} \mathrm{Rb}$.

\section{Soliton dynamics in an optical lattice and sound emission}

We first consider the behaviour of a dark soliton under harmonic confinement only, in order to compare and contrast this to the behaviour observed when an optical 

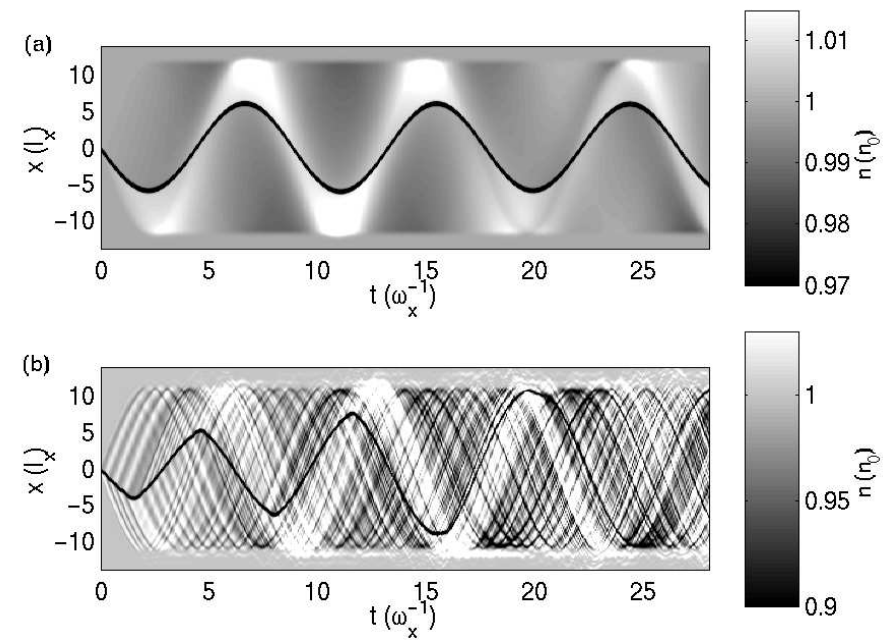

Figure 2. Space-time carpet plots of the renormalised condensate density (actual density minus background density) for (a) harmonic confinement only and (b) the same harmonic trap plus an optical lattice with $V_{0}=0.2 \mu$ and $\lambda=2.4 l_{x}$. Both cases start with a dark soliton at the origin with speed $v=0.5 c$. Sound waves are clearly emitted by the dynamically unstable soliton, not only as it climbs the trap walls but also as it traverses the lattice sites. Note that the sound waves in (b) have a much greater amplitude (see the different density scales).

lattice is additionally imposed. Figure 2(a) shows a space-time carpet plot of the renormalised condensate density (actual density minus background density) of a dark soliton, initially at the origin with speed $v=0.5 c$. The soliton oscillates back and forth in the trap at a rate roughly equal to $\omega_{s}=\omega_{x} / \sqrt{2}$, as expected [10, 22, 23], 33. As it climbs the side of the trap, passing through the inhomogeneous background density, it emits counter-propagating sound waves of opposing amplitude, and becomes asymmetrically deformed [25]. The sound reflects off the edge of the condensate and subsequently reinteracts with the soliton around $t \sim 5 \omega_{x}^{-1}$. An equilibrium is rapidly established where the soliton, on average, reabsorbs as much sound as it emits. Hence the soliton energy features oscillations due to the emission/reabsorption process, but with no net decay 24. The soliton position and energy as a function of time are shown respectively by grey lines in figure $3(\mathrm{a})$ and (b).

Consider now the case of imposing, in addition to the harmonic confinement, an optical lattice with height $V_{0}=0.2 \mu$ and wavelength $\lambda=2.4 l_{x}$, as shown in figure 2(b). The presence of the optical lattice causes the soliton dynamics to differ significantly from the pure harmonic case. As the soliton oscillates, it not only climbs the walls of the harmonic trap, but also traverses the lattice bumps. It thereby experiences increased dynamical instability and emits additional sound waves as it passes over each lattice site. The harmonic trap still contributes to the sound emission, as seen by the faint 'white' band of sound in figure 2(b), but this emission is heavily obscured (compared to figure 2(a)) by the lattice-generated sound waves. Importantly, in this case, reflection of sound from the edge of the condensate no longer leads to the establishment of an equilibrium between sound emission and reabsortion. This is because the presence of the optical lattice dephases, or mixes, the emitted sound such that only a proportion is reabsorbed. The soliton becomes faster and shallower, and 


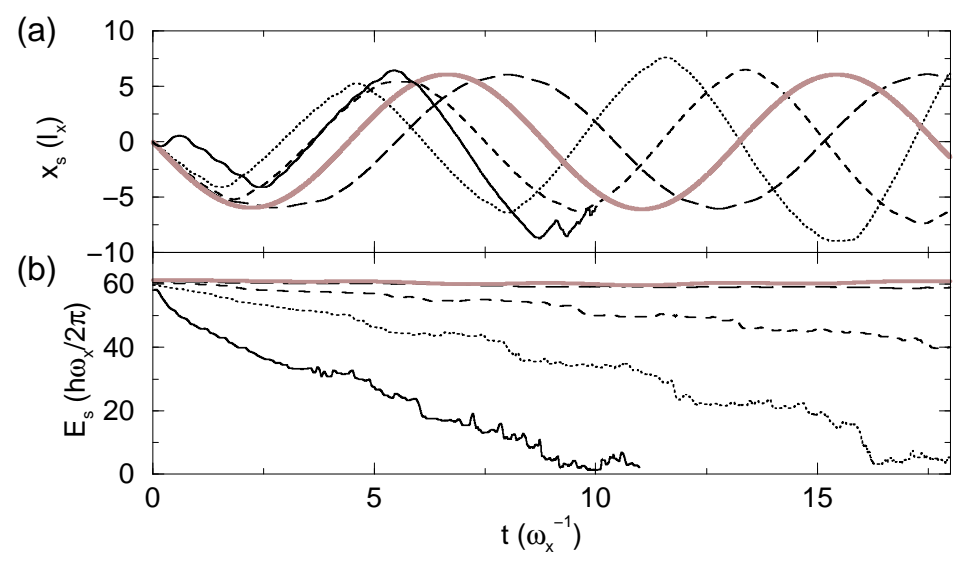

Figure 3. Effect of lattice height on the soliton dynamics. (a) Path and (b) energy of the soliton in a harmonic trap (solid grey lines), and a harmonic trap plus an optical lattice with $\lambda=2.4 l_{x}$ and lattice heights $V_{0}=0.05 \mu$ (long-dashed line), $V_{0}=0.1 \mu$ (dashed line), $V_{0}=0.2 \mu$ (dotted line) and $V_{0}=0.4 \mu$ (solid line).

'anti-damps' to larger amplitudes. By $t \sim 20 \omega_{x}^{-1}$ the soliton has becomes so fast and shallow that it is virtually indistinguishable from, and gradually merges into, the sound field. The soliton position and energy as a function of time are shown respectively by dotted lines in figure 3(a) and (b).

\section{Effect of varying the lattice height}

We now consider the effect on the soliton dynamics of varying the height of the optical lattice $V_{0}$. Throughout this section, the periodicity of the lattice is kept constant at $\lambda / 2=1.2 l_{x}$. Figure 3 shows the soliton position and energy as a function of time for various lattice heights. The soliton energy $E_{s}$ is calculated by integrating the GP energy functional,

$$
\varepsilon(\psi)=\frac{\hbar^{2}}{2 m}|\nabla \psi|^{2}+V|\psi|^{2}+\frac{g}{2}|\psi|^{4},
$$

across the 'soliton region', conveniently defined to be $\left(x_{s} \pm 5 \xi\right)$, and subtracting from this the corresponding contribution of the background fluid (obtained from the timeindependent solution of equation (1) in the absence of a soliton).

Figure 3 shows soliton oscillations and energies for various lattice heights. Increasing the lattice height leads to two distinct effects which modify the soliton oscillations in opposing manners. Firstly, the optical lattice modifies the condensate profile, periodically reducing the density. This inhibits the motion of the soliton and tends to shift its turning point towards the centre of the trap and increase its oscillation frequency. This effect can be seen by the dashed line $\left(V_{0}=0.1 \mu\right)$ in figure 3(a). Indeed, if the lattice height is sufficiently large, the soliton will initially be trapped within a single lattice site. Secondly, the optical lattice induces sound emission from the soliton. The soliton energy decays essentially monotonically, with the average decay rate increasing with lattice height. This decay leads to a gradual decrease in the oscillation frequency and increase in the amplitude. For small lattice 

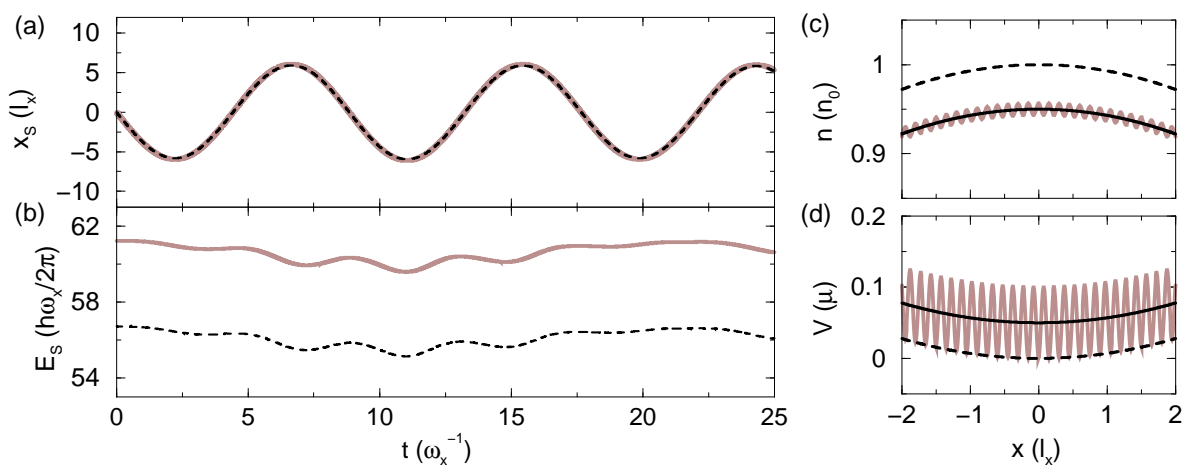

Figure 4. Effect of small lattice periodicity, $\lambda / 2 \sim \xi$ (or less), compared to no optical lattice. (a) Path and (b) energy of a dark soliton in a tight optical lattice, $\lambda=0.3 l_{x}(2.5 \xi)$, with height $V_{0}=0.1 \mu$ (dashed line), compared to the absence of the optical lattice (grey line). (c) The density (grey line) for this tight lattice cannot fully heal, shifting the average density down (bold line) in relation to the harmonic trap density (dashed line). (d) Since the fluid cannot heal fully to the tight lattice (grey line), the effective potential tends towards a harmonic trap shifted upwards (solid line) by the mean lattice potential $V_{0} / 2$. The harmonic potential is also shown (dashed line).

heights, e.g. $V_{0}=0.05 \mu$ (long-dashed line in figure $3(\mathrm{a})$ and (b)) the optical lattice poses little resistance to the soliton motion, and the low level of sound emission it induces causes the main observable effects. These are a small decrease in the soliton oscillation frequency and a slow decay of the soliton energy. The competing effects are most clearly observed for $V_{0}=0.4 \mu$ (solid line in figure 3(a)). The soliton is initially confined to a single lattice site, with a high oscillation frequency. Sound emission causes the soliton to gradually 'anti-damp' out of this site into adjacent sites, leading to a decrease in its oscillation frequency and an increase in its amplitude. This effect has already been reported in [27. The soliton rapidly accelerates towards the edge of the cloud where it ultimately merges into the sound field. However, in the limit $V_{0}>\mu$, an array of mini-condensates is formed. Each lattice site becomes an effectively closed system, and the emitted sound cannot escape the site. In this limit the GP equation predicts that full sound reabsorption by the soliton occurs, which stabilises it against decay, as already observed in 24. In the limit of very small lattice periodicities, the discrete nonlinear Schrodinger equation may be a more appropriate description, in which case additional oscillatory instabilities come into play [34, 26].

\section{Effect of varying the lattice periodicity}

We next consider how the periodicity of the lattice affects the soliton dynamics, an effect also considered analytically and numerically in 27. We start by discussing an anomalous regime, the tight lattice regime, where the lattice periodicity is of the order of, or less than, the soliton width. The path and energy of a dark soliton with initial speed $v=0.5 c$ under the influence of a tight optical lattice $\left(\lambda=0.3 l_{x}(2.5 \xi)\right.$ and $V_{0}=0.1 \mu$ ) are shown by the dashed line in figure 4(a) and 4(b) respectively. The path of the soliton is identical to that in the absence of the optical lattice (solid grey line), while the energy has the same form but is shifted to a lower value. This effect 

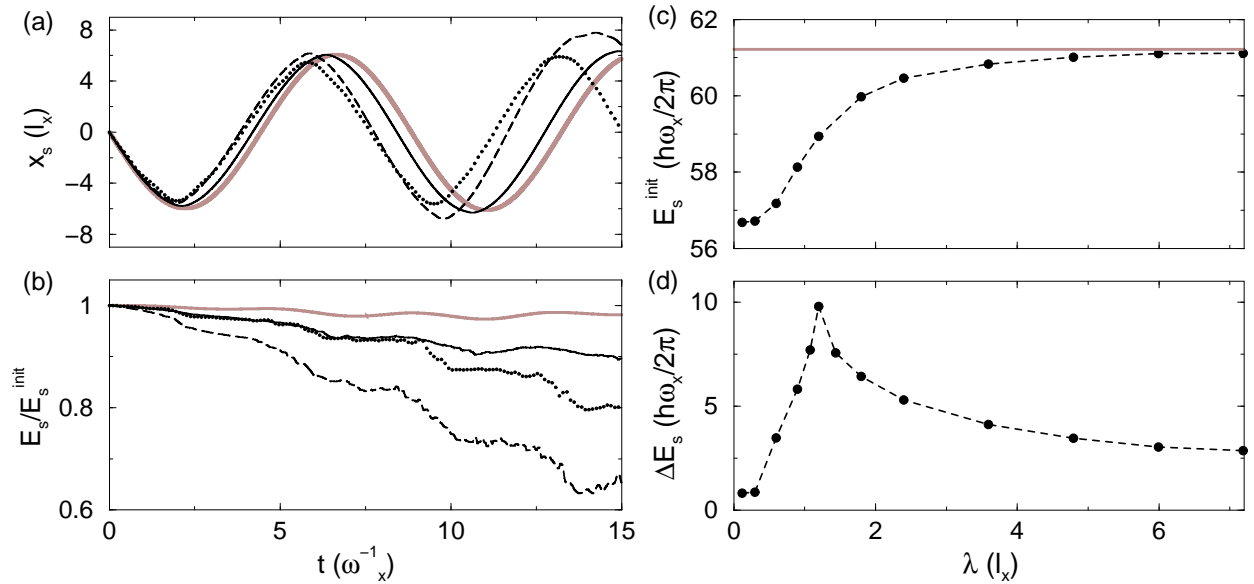

Figure 5. Effect of lattice periodicity on the soliton dynamics. (a) Path and (b) energy, rescaled by the initial soliton energy $E_{s}^{\text {init }}$, of a soliton in a harmonic trap (grey line) and an additional optical lattice with height $V_{0}=0.1 \mu$ and various

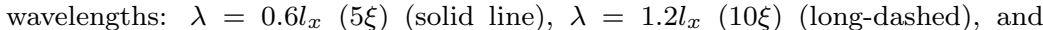
$\lambda=4.8 l_{x}(40 \xi)$ (dotted line). (c) Initial soliton energy $E_{s}^{\text {init }}$ as a function of lattice wavelength, compared to the $V_{0}=0$ harmonic case (horizontal grey line). (d) Loss of soliton energy $\Delta E_{s}$ after one full oscillation, as a function of lattice wavelength.

can be explained by careful consideration of the density perturbations caused by the optical lattice. In the limit where the lattice wavelength is of the order of, or less than, the soliton width, the fluid cannot heal to the optical lattice, and the associated density modulations smear together. Then the background density becomes reduced across the whole condensate, and in particular the peak density, $n_{0} \rightarrow n_{0}^{\prime}$. This is due to the fact that, in our simulations, the chemical potential $\mu$ is fixed. If, however, the peak density/number of particles were kept fixed, then the chemical potential would increase accordingly. The soliton essentially sees a constant effective optical lattice potential of height $V_{0} / 2$, and overall it sees the ambient harmonic potential shifted upwards by this amount, an effect illustrated in figure 4(c) and 4(d). In both cases of a pure harmonic trap and a harmonic trap modified by a tight optical lattice, the initial soliton speed has been set to the same value $v=0.5 c$ (i.e. the dependence on peak density is removed), ensuring that the paths are identical. If we assume a ThomasFermi relationship between potential and density, $n(x)=n_{0}-V(x)$, then we would expect, to first order, the peak density to be decreased by $\left(V_{0} / 2\right) n_{0}=0.05 n_{0}$. Equation 4 implies that the initial soliton energy will be rescaled by a factor of $\left(n_{0}^{\prime} / n_{0}\right)^{\frac{3}{2}}$, i.e. $E_{s}^{\text {init }} \rightarrow 0.926 E_{s}^{\text {init }}$, and this is indeed the modified soliton energy observed.

We now investigate the effect of lattice periodicity on the soliton dynamics. The soliton path and energy for various lattice wavelengths are shown in figure 5(a) and (b) respectively. In order to remove the effect of reduced peak density for sufficiently tight lattices (based on our fixed chemical potential simulations), the soliton energies in figure 5(b) have been rescaled in terms of the initial soliton energy. This effect is quantified in figure 5(c) which shows the variation in the initial soliton energy as a function of lattice wavelength. 
Looking now at the decay of the (normalised) soliton energy as a function of time for various lattice wavelengths, we find that increasing the lattice wavelength (but still remaining far from the tight lattice regime considered earlier) leads to the decay of the soliton energy and an associated increase in the soliton amplitude and frequency (e.g. $\lambda=0.6 l_{x}$, solid line in figure $5(\mathrm{a})$ and(b)) in relation to the pure harmonic case (grey line). The rate of this decay increases up to some resonant wavelength $\left(\lambda=1.2 l_{x}\right.$, dashed line). Any further increase in the wavelength leads to a slowing of the decay. This is illustrated in figure 5(b) by the reduced decay rate for $\lambda=4.8 \xi$ (dotted line) compared to $\lambda=1.2 \xi$ (dashed line).

The dependence of the soliton decay on the lattice periodicity can be explained in terms of the density inhomogeneity that the soliton experiences. To illustrate this analysis more quantitatively, figure 5(d) plots the loss in soliton energy after one full oscillation as a function of lattice wavelength. For tight lattices, where the lattice periodicity is of the order of, or less than, the soliton width, the soliton effectively sees a displaced harmonic trap, and so experiences no net decay, due to complete sound reabsorption. In this limit, the soliton energy oscillates periodically due to the continuous sound emission/reabsorption process. This takes the form a beating effect caused by the different oscillation frequencies of the soliton and sound (dipole mode) 24]. Therefore, one full oscillation of the soliton in the trap does not correspond to one beat period, and hence the soliton energy has not yet returned to its initial value, explaining the small apparent energy loss. For progressively weaker lattices, the density begins to heal to the optical lattice, thereby modifying the condensate density from the harmonic trap profile. This increases the density inhomogeneity that the soliton sees, and induces an increase in the rate of decay. Furthermore, the presence of these density modulations dephases the sound field such that the soliton is no longer fully stabilised by sound reabsorption.

Increasing the lattice periodicity further leads to some critical wavelength the condensate density can heal fully to the lattice potential. This represents the system with the maximum density inhomogeneity (for a fixed lattice height) and therefore features the most rapid soliton decay. This point is indicated by the resonance in

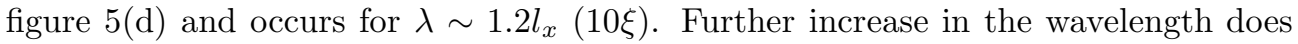
not change the depth of the density modulations, but merely stretches them out. This now reduces the density gradient experienced by the soliton [25] and causes a reduction in the decay rate. As $\lambda \rightarrow \infty$ the decay will asymptotically tend towards zero as the trap potential returns to becoming effectively harmonic.

\section{Quantifying the sound emission}

We will now quantatively discuss the soliton dynamics up until the time when the sound reinteracts with the soliton. Figure 6 shows the short-term dynamics of a dark soliton in the optical lattice geometry considered in Section $3\left(V_{0}=0.2 \mu, \lambda=2.4 l_{x}\right.$, dotted line of figure 3), along with the corresponding dynamics in the absence of the optical lattice (grey line of figure 3). The soliton motion (figure 6(a)) is clearly inhibited by the modified local density (figure 6(b)), causing it to turn around earlier and at a lower amplitude than in the absence of the optical lattice. The soliton accelerates and decelerates periodically as it traverses the lattice sites, as shown in figure 6(c) (solid line, left axis) with the oscillation weakly modulated by the additional acceleration induced by the ambient harmonic trap (grey line in same figure). This contribution is zero when the soliton is at the centre of the trap and a maximum when 


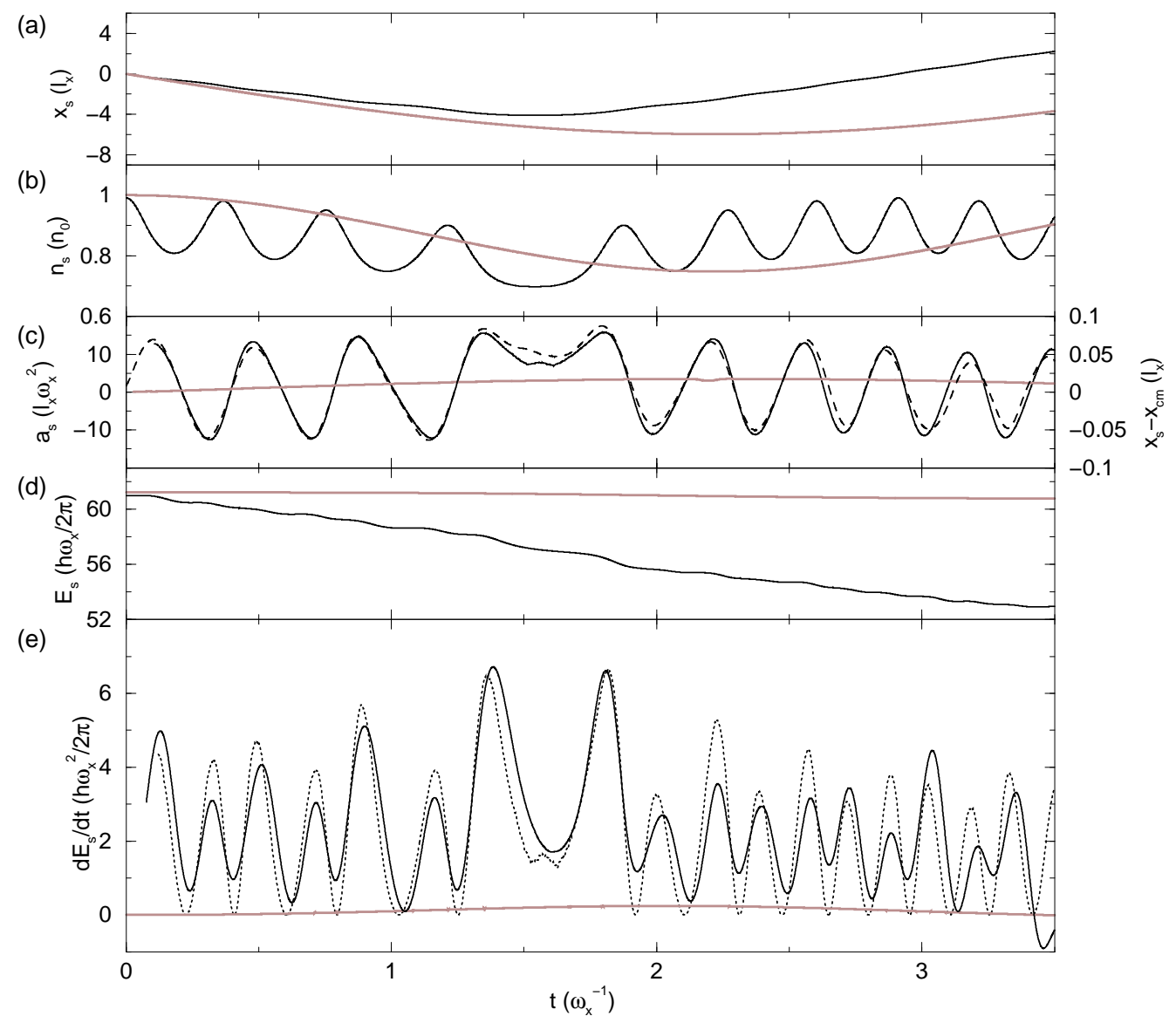

Figure 6. Comparison of dynamics of a soliton with initial speed $0.5 c$ in a harmonic trap plus optical lattice with $V_{0}=0.2 \mu$ and $\lambda=20 \xi$ (black line) and corresponding dynamics only in the presence of the same harmonic trap (grey lines). (a) Path of soliton versus time. (b) Background density at the soliton position. (c) Soliton acceleration (bold line, left axis) and deformation parameter $\left(x_{s}-x_{\mathrm{cm}}\right)$ (dashed line, right axis). (d) Soliton energy. (e) Power emitted by the soliton as computed from the GP energy functional (solid line), compared with the acceleration squared law of equation $(7)$ with $\kappa=1.8\left(\hbar / l_{x}\right)$ (dotted line).

the soliton is at an extremum.

The emission of oppositely "charged" sound waves from the soliton leads to an asymmetric distortion of the soliton profile, and a shift of the soliton centre of mass $x_{\mathrm{cm}}$ from the density minimum $x_{s}$, where the soliton centre of mass is defined as,

$$
x_{\mathrm{cm}}=\frac{\int_{s} x\left(|\psi|^{2}-n\right) \mathrm{d} x}{\int_{s}\left(|\psi|^{2}-n\right) \mathrm{d} x} .
$$

This shift, shown in figure 6(c) (dashed line, right axis), is directly proportional to the soliton acceleration (solid line, left axis), in accordance with earlier findings 25].

The motion of the soliton through the lattice causes a periodic cascade of the soliton energy (figure 6(d)). This cascade is more evident when considering the power 
emitted by the soliton, shown in figure 6(e). The power consists of a series of peaks of various amplitudes, which clearly occur whenever the soliton traverses the side of a lattice site, i.e. where the density gradient is a local maximum. The peaks are greatest at around $t \sim 1.5 \omega_{x}^{-1}$, which corresponds to the point of maximum soliton acceleration. Indeed, the power emission can be well fitted by an acceleration-squared law,

$$
\frac{d E}{d t}=-\kappa a^{2}
$$

where $\kappa$ is a constant coefficient $\left(\kappa=0.0255\left(\hbar / l_{x}\right)\right.$ in figure $\left.6(\mathrm{e})\right)$. This is also in agreement with an acceleration-squared power law derived analytically for dynamically unstable dark solitons in the context of nonlinear optics [35. Although [35] deals with a homogeneous system in which the instabilities arise from imperfections in the medium, such an approach has recently been considered for dark solitons in inhomogeneous condensates [24, 25]. When the emitted sound reinteracts with the soliton $\left(t>3 \omega_{x}^{-1}\right)$, it is partially reabsorbed (as evident from the negative values acquired by the power curve of figure $6(\mathrm{e})$ ), and equation (7) ceases to be valid. For the same reasons, the proportionality between acceleration and the deformation parameter is no longer valid.

\section{Conclusions}

We have made a quantitative investigation of the dynamics of a dark soliton induced by the presence of an optical lattice. We find, in general, that the optical lattice significantly affects the motion and dynamical stability of the soliton. The exception to this is in the tight lattice regime where the fluid cannot heal to the lattice and the soliton effectively sees a harmonically trapped condensate.

By considering optical lattices of varying depths and wavelengths we have illustrated the sensitive dependence of the soliton dynamics on these parameters, and explained the dependence in terms of the density inhomogeneity experienced by the soliton. The effect of the optical lattice, and the associated density modulations, is to create extra dynamical instability in the soliton, in addition to that caused by the ambient harmonic trap. This induces soliton decay via the emission of sound waves. For deep and moderately tight lattices this effect is considerable: the soliton rapidly decays, accelerating to the edge of the condensate, where it disappears into the sound field. Unlike in a pure harmonic trap, the presence of the optical lattice tends to modify the emitted sound such that the full stabilisation of the soliton by sound reabsorption is prevented.

The soliton-sound interaction in an optical lattice geometry can be experimentally probed in a quasi-one-dimensional condensate, providing an alternative method to that suggested in 24. The dynamical dissipation is observable as a change in the soliton amplitude and oscillation frequency, by repeated expansion of the cloud and subsequent absorption imaging, after a variable evolution time in the trap. The timescales considered in this work are consistent with the expected thermodynamic soliton lifetime of $1 \mathrm{~s}$ [10. The intensity of the laser beams that form the optical lattice controls the lattice height, while varying the relative angle between the beams allows the full range of lattice periodicities considered here to be realised. Modification of these two parameters enables full experimental control of the dynamical instability of the soliton induced by the optical lattice. 
Dark soliton in a quasi-1D BEC perturbed by an optical lattice

\section{Acknowledgments}

We acknowledge financial support from the UK EPSRC and discussions with D. J.

Frantzeskakis and P. G. Kevrekidis.

\section{References}

[1] Greiner M, Mandel O, Esslinger T, Hansch T W and Bloch I 2002 Nature 41539

[2] Anderson B P and Kasevich M A 1998 Science 2821686

[3] Morsch O, Muller J H, Cristiani M, Ciampini D and Arimondo E 2001 Phys. Rev. Lett. 87 140402

[4] Orzel C, Tuchman A K, Fenselau M L, Yasuda M and Kasevichm M A 2001 Science 2912386

[5] Cataliotti F S, Burger S, Fort C, Maddaloni P, Minardi F, Trombettoni A, Smerzi A and Inguscio M 2001 Science 293843

[6] Burger S et al 1999 Phys. Rev. Lett. 835198

[7] Denschlag J et al 2000 Science $\mathbf{2 8 7} 97$

[8] Dutton Z, Budde M, Slowe C and Hau L V 2001 Science 293663

[9] Kivshar Y S and Luther-Davies B 1998 Phys. Rep. 27881

[10] Fedichev P O, Muryshev A E and Shlyapnikov G V 1999 Phys. Rev. A 603220

[11] Dziarmaga J, Karkuszewski Z P and Sacha K 2003 J. Phys. B 361217

[12] Feder D L, Pindzola M S, Collins L A, Schneider B I and Clark C W 2000 Phys. Rev. A 62 053606

[13] Brand J and Reinhardt W P 2002 Phys. Rev. A 65043612

[14] Muryshev A et al 2002 Phys. Rev. Lett. 89110401

[15] Anderson B P et al 2001 Phys. Rev. Lett. 862926

[16] Mamaev A V, Saffman M and Zozulya A A 1996 Phys. Rev. Lett. 762262

[17] Goerlitz A et al 2001 Phys. Rev. Lett. 87130402

[18] Schreck F et al 2001 Phys. Rev. Lett. 87080403

[19] Ott $\mathrm{H}$ et al 2001 Phys. Rev. Lett. 87230401

[20] Haensel W et al Nature $\mathbf{4 1 3} 498$

[21] Muryshev A E, van Linden van den Heuvell H B and Shlyapnikov G V 1999 Phys. Rev. A 60 R2665

[22] Busch T and Anglin J R 1999 Phys. Rev. Lett. 842298

[23] Huang G, Szeftel J and Zhu S 2002 Phys. Rev. Lett. 65053605

[24] Parker N G, Proukakis N P, Leadbeater M and Adams C S 2003 Phys. Rev. Lett. 90220401

[25] Parker N G, Proukakis N P, Leadbeater M and Adams C S 2003 J. Phys. B 362891

[26] Kevrekidis P G, Carretero-Gonzalez R, Theocharis G, Frantzeskakis D J and Malomed B A 2003 Phys. Rev. A 68035602

[27] Theocharis G, Frantzeskakis D J, Kevrekidis P G, Carretero-Gonzalez R and Malomed B A 2003 Preprint

[28] Denschlag J H et al 2002 J. Phys. B 353095

[29] Kevrekidis P G, Frantzeskakis D J, Malomed B A, Bishop A R and Kevrekidis I G 2003 New J. Phys. 564

[30] Jackson A D, Kavoulakis G M and Pethick C J 1998 Phys. Rev. A 582417

[31] Morgan S A, Ballagh R K and Burnett K 1997 Phys. Rev. A 554338

[32] Reinhardt W P and Clark C W 1997 J. Phys. B 30 L785

[33] Frantzeskakis D J, Theocharis G, Diakonos F K, Schmelcher P and Kivshar Y S 2002 Phys. Rev. A 66053608

[34] Johansson M and Kivshar Y S 1999 Phys. Rev. Lett. 8285

[35] Pelinovsky D E, Kivshar Y S and Afanasjev V V 1996 Phys. Rev. E 542015 